\title{
Experimental validation of TMVR technologies: a bumpy road with the risk of getting lost in translation
}

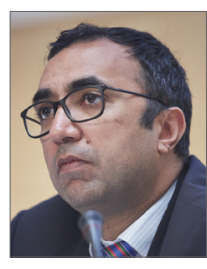

Azeem Latib $^{1,2}$, MD; Juan Granada ${ }^{3}$, MD

1. Interventional Cardiology Unit, San Raffaele Scientific Institute, Milan, Italy; 2. Interventional Cardiology Unit, EMO-GVM

Centro Cuore Columbus, Milan, Italy; 3. Cardiovascular Research Foundation, Columbia University, New York, NY, USA

Mitral regurgitation (MR) is the most frequent valye pathology in Europe and the USA with moderate-to-severe MR occurring in almost 1 in 10 people aged 75 or older ${ }^{1}$. At least $50 \%$ of patients with symptomatic severe MR are treated medically and not offered any surgical mitral options due to comorbidities and high surgical risk profile ${ }^{2,3}$. The prognosis in medically managed patients with severe MR is poor with a $20 \%$ mortality at one year, a $50 \%$ mortality at five years, and $90 \%$ of surviving patients being hospitalised for heart failure within five years from diagnosis ${ }^{3}$. This unmet clinical need has led to the development of over 70 transcatheter-based technologies aimed at either repairing or replacing the mitral valve. This large number of devices and technological approaches has resulted from the need to develop solutions to a condition with a high degree of anatomical variability and the technical desire to replicate proven surgical techniques.

Mitral valve disease is very complex and involves not only the annulus and leaflets but also surrounding subvalvular, aortic and myocardial structures. The mitral annulus is asymmetric and frequently gets deformed from its original saddle shape as the disease progresses. It is also a very dynamic structure lacking structural support, thus making the anchoring of sutureless devices more challenging. The mitral leaflets are irregular in size and geometry; the subvalvular apparatus is very complex and highly variable between patients. Also, the closing valve forces are high, requiring the use of durable leaflet materials to ensure long-term durability ${ }^{4}$. Furthermore, the geometry of the mitral valve apparatus and left ventricle can vary considerably between patients with the same aetiology of MR, between different aetiologies of MR and even in the same patient as the disease progresses over time.

Despite the broad enthusiasm and substantial investment, significant challenges exist in the transcatheter mitral valve replacement (TMVR) field ${ }^{5}$. First, due to the complex biomechanical forces involved in ventricular contraction and annular deformation, long-term metallic frame and leaflet durability becomes a challenge. Second, due to the multiple design features and high radial strength required, these devices still display high crossing profiles (above 32 Fr). Third, depending on the TMVR design, specific complex device orientation, fixation and deployment techniques

*Corresponding author: Interventional Cardiology Unit, San Raffaele Scientific Institute, Via Olgettina 60, 20132 Milan, Italy. E-mail: alatib@gmail.com 
are usually required. Fourth, personalised device-guided sizing algorithms still reject a large number of patients due to anatomical variations, sizing considerations or potential for LVOT obstruction. Finally, the prothrombogenic profile of these devices is still unknown.

TMVR may have potential advantages over transcatheter repair in that a single device may be used regardless of the type of primary pathology and could potentially deliver reproducible and predictable elimination of MR. However, most of the early clinical experience has been limited to the transapical approach in very high-risk or prohibitive-risk patients, resulting in a higher risk of periprocedural complications and 30-day mortality. On the other hand, transcatheter mitral repair has been associated with a better safety profile and high procedural success rates without negatively impairing ventricular geometry and function; however, it requires a steep learning curve and may require a knowledge of multiple devices in order to guarantee surgical-like results. Considering the complexity of the mitral valve anatomy and pathological variations seen during the course of disease progression, it is unlikely that a single device will fulfil all the clinical and technical requirements to treat these complex patients. Thus, the interventional treatment of mitral valve disease will probably require a lesionspecific "toolbox" approach, potentially including percutaneous repair, replacement and ventricular support.

In the current issue of EuroIntervention, the experimental data of the novel NaviGate ${ }^{\mathrm{TM}}$ TMVR system (NaviGate Cardiac Structures, Inc., Lake Forest, CA, USA) are presented ${ }^{6}$. The NaviGate is a low-profile ( $\sim 21 \mathrm{~mm}$ height) self-expanding nitinol frame device including three equine pericardial leaflets and a conical configuration with larger diameter towards the ventricle. The valve anchors to the mitral annulus using 12 radially arranged winglets on the atrial side and 12 radially arranged graspers on the ventricular side that grasp and penetrate the leaflets and subvalvular structures.

\section{Article, see page 1401}

The valve is available in five sizes (from $36 \mathrm{~mm}$ to $52 \mathrm{~mm}$ ) and can be delivered using the antegrade and retrograde approach via a $32 \mathrm{Fr}$ delivery system. In this study, the NaviGate valve achieved a high periprocedural device success rate $(\sim 83 \%)$ with the animal surviving longest in the chronic study only surviving to 54 days. Overall, valve frame stability and haemodynamics remained stable over time, resulting in low transvalvular gradients, no cases of LVOT obstruction and no residual MR in more than two thirds of the animals studied. Residual MR when present was mild and more often central than paravalvular, which raises questions about the radial strength of the device and leaflet coaptation issues related to frame deformation following device implantation. Although macroscopic findings suggested proper tissue ingrowth and lack of thrombus formation over time, the degree of device-induced injury on the subannular structures was not described in detail.

Currently, the main reasons for patients not being accepted for TMVR include the risk of LVOT obstruction and sizing mismatch. The NaviGate valve may be partially able to address these issues because of its low profile and larger size range. Also, the valve has a low atrial footprint which may potentially decrease thrombogenicity. However, long-term durability data of equine pericardial leaflets are still under investigation and long-term animal data on the durability of the valve and interaction of the anchoring mechanism with the underlying tissue are still needed. As with other devices, the biggest translational challenge will be to adapt the technology to a lower-profile transseptal system. Considering the challenges of transseptal TMVR, this valve may be better suited for transcatheter tricuspid valve replacement in which the technical device requirements are less demanding compared to mitral device technologies.

Due to the nature of the animal models and experimental settings, the experimental validation of TMVR devices is challenging and can encounter fundamental differences compared to the human environment. First, due to animal size considerations, only small size $(<40 \mathrm{~mm})$ TMVR devices can be tested in typical animal models. Second, the mitral valve shape is typically more oval, inducing a high degree of valve deformation compared to humans. Third, the ventricle is smaller and hyperdynamic, inducing higher stress to the implanted valve. Fourth, the surrounding tissue support is healthy and fragile. Fifth, the thrombogenic profile is different, and clot formation is not frequently seen in large animal models. Then, as has occurred with other technologies (i.e., TAVR), experimental findings have not been able to predict device performance fully in the clinical setting.

We congratulate the authors and inventors for publishing and sharing their experimental findings with the scientific community and would encourage others to do the same. Understanding the nature of these findings is an important step not only to appreciating the biological performance of these devices but also to helping to define the potential regulatory pathways and training needs of physicians involved in FIH studies. However, as has happened with other technologies in the past, investigators and regulatory agencies must be prepared to understand the potential impact of animal model-specific findings on clinical device use.

The comprehensive preclinical evaluation of TMVR devices is key for the development of this field. Customised human-like sizing algorithms and procedural development can be accomplished in the experimental setting. Vascular access safety, acute and longterm device-derived injury can also be tested in animals. It is also important that proper sample size and follow-up endpoints be used before moving into FIH clinical studies. We would strongly recommend that physicians and inventors developing TMVR systems consult the "ANSI/AAMI/ISO 5840-3: 2013" document prior to designing their preclinical strategies?

Since there are currently more than 30 TMVR systems under development and at least eight have already been implanted in humans $s^{5}$, it does beg the question as to whether we really need another TMVR device. Currently, the largest clinical experience is with the Intrepid ${ }^{\mathrm{TM}}$ (Medtronic, Inc., Redwood City, CA, USA) and Tendyne (Abbott Vascular, Santa Clara, CA, USA) TMVR systems. Both devices continue slow enrolment of highly selected patients and have been able to improve both periprocedural success 
rates and 30-day clinical outcomes ${ }^{8}$ (Muller D. TENDYNE: 1-Year Outcomes of Transcatheter MV Replacement in Patients With Severe Mitral Regurgitation. Presented at Transcatheter Cardiovascular Therapeutics [TCT] 2017 in Denver on 1 November 2017. Available at: https://www.tctmd.com/slide/tendyne-1-yearoutcomes-transcatheter-mv-replacement-patients-severe-mitralregurgitation. Accessed 19 November 2017). In order to be successful, emerging TMVR systems will have to improve device features, ease of use and procedural steps. More importantly, they need to decrease drastically the high rejection rate due to anatomical considerations. Other desirable features include minimal interference with the subvalvular apparatus, lower risk of LVOT obstruction, and possibly the ability to reposition and re-sheath the valve. The transseptal route of implantation promises to disrupt the TMVR market and has the potential to simplify TMVR procedures and gain access to a different patient population. In the early phases of TMVR development, preclinical and clinical data have been fundamental in improving patient selection, implantation technique and short-term clinical outcomes. The successful validation process of TMVR devices has to follow a thoughtful step-bystep approach. The road to clinical success will very likely be long and bumpy and we need to ensure that translational data gathered from early clinical experience do not get lost in translation.

\section{Conflict of interest statement}

A. Latib is a consultant for Mitralign, Millipede, Mitraltech, InnovHeart, Medtronic, and Abbott Vascular. J. Granada is a co-founder of Cephea. The CRF-Skirball Center for Innovation has received research grants from Abbott Vascular, Edwards, Medtronic, Mitralign, and NaviGate Cardiac Structures.

\section{References}

1. Nkomo VT, Gardin JM, Skelton TN, Gottdiener JS, Scott CG, Enriquez-Sarano M. Burden of valvular heart diseases: a population-based study. Lancet. 2006;368:1005-11.
2. Mirabel M, Iung B, Baron G, Messika-Zeitoun D, Détaint D, Vanoverschelde JL, Butchart EG, Ravaud P, Vahanian A. What are the characteristics of patients with severe, symptomatic, mitral regurgitation who are denied surgery? Eur Heart J. 2007;28: 1358-65.

3. Goel SS, Bajaj N, Aggarwal B, Gupta S, Poddar KL, Ige M, Bdair H, Anabtawi A, Rahim S, Whitlow PL, Tuzcu EM, Griffin BP, Stewart WJ, Gillinov M, Blackstone EH, Smedira NG, Oliveira GH, Barzilai B, Menon V, Kapadia SR. Prevalence and outcomes of unoperated patients with severe symptomatic mitral regurgitation and heart failure: comprehensive analysis to determine the potential role of MitraClip for this unmet need. J Am Coll Cardiol. 2014;63: 185-6.

4. Regueiro A, Granada JF, Dagenais F, Rodes-Cabau J. Transcatheter Mitral Valve Replacement: Insights From Early Clinical Experience and Future Challenges. J Am Coll Cardiol. 2017;69:2175-92.

5. Patel A, Bapat VN. Transcatheter mitral valve replacement: device landscape and early results. EuroIntervention. 2017;13: AA31-9.

6. Navia JL, Baeza C, Maluenda G, Kapadia S, Elgharably H, Sadowski J, Bartuś K, Beghi C, Thyagarajan K, Bertwell R, Quijano RC. Transcatheter mitral valve replacement with the NaviGate stent in a preclinical model. EuroIntervention. 2017; 13:e1401-9.

7. ISO 5840-3:2013. Cardiovascular implants -- Cardiac valve prostheses -- Part 3: Heart valve substitutes implanted by transcatheter techniques. Available at: https://www.iso.org/standard/51313.html (accessed 19 November 2017).

8. Bapat V, Rajagopal V, Meduri C, Farivar RS, Walton A, Duffy SJ, Gooley R, Almeida A, Reardon MJ, Kleiman NS, Spargias K, Pattakos S, Ng MK, Wilson M, Adams DH, Leon M, Mack MJ, Chenoweth S, Sorajja P; Intrepid Global Pilot Study Investigators. Early Experience with New Transcatheter Mitral Valve Replacement. J Am Coll Cardiol. 2017 Oct 25. [Epub ahead of print]. 